\title{
Designing Experimental Procedures: A Low Cost, Hands On Project that Students Actually Enjoy
}

\author{
Capt Shad Reed, MAJ Donna Dorminey \\ United States Military Academy, West Point
}

\begin{abstract}
Because many students are unfamiliar with the development of experimental procedures, a project was sought that would allow students to use the design process to generate an optimal experimental procedure. An experiment that required students to determine the coefficient of drag for a small, dollar-store parachute figurine was selected. After a brief introduction to the pertinent theory, students were tasked to determine a governing equation for the experiment, identify measurable variables, generate concepts for experimental procedures, evaluate them based on customer requirements, conduct the experiment, analyze the results, and suggest improvements for subsequent iterations. Survey data indicates that most students enjoyed the project even though it required hard work, and that most learned how to design an optimal experimental procedure.
\end{abstract}

Introduction

Practicing engineers are often forced to answer complex questions by conducting an experiment and analyzing data. However before the experiment can be conducted, the engineer must devise an experimental procedure that will yield accurate results and not require an excessive expenditure of resources. The ability to develop an appropriate experimental procedure is so vital that the Accreditation Board for Engineering and Technology (ABET) requires that, "Engineering programs must demonstrate that their graduates have an ability to design and conduct experiments" .

Given the number of experiments conducted throughout their undergraduate studies, most students that have graduated are well prepared to conduct an experiment that has an established set of experimental procedures. Unfortunately, students are not nearly as well prepared to design experimental procedures. Occasionally, laboratory exercises require students to develop experimental procedures. But even this requirement fails to satisfy the criterion set forth by ABET because design includes both the generation and evaluation of several potential solutions. Indeed, it is rare that students are ever required to design experimental procedures.

\section{Background}

With varying degrees of success, the Fluid Mechanics course at the United States Military Academy has traditionally been tasked to formally address the design of an experiment. This is somewhat challenging given that the course is one of the first engineering courses taken by cadets and is typically taken during the third year by both engineering and non-engineering majors. Further, it is highly unusual that any of the cadets have been introduced to a formal design process.

\section{Proceedings of the 2002 American Society for Engineering Education Annual Conference \& Exposition}

Copyright $\odot$ 2002, American Society for Engineering Education 
Several of the previous projects that were used to address the issue of designing experimental procedures were theoretical exercises that focused on the development of an experimental procedure as opposed to the design of an experimental procedure. For instance, the previous project required cadets to develop an experiment that could be used to determine the viscosity of a fluid. While this project had some redeeming qualities, it did not illustrate that an experimental procedure can be designed in a manner not that dissimilar to an aircraft or an automobile. Additionally, students expressed the desire for the projects to be more interesting and hands on.

\section{Design of an Experiment}

Rather than attempting to fix an existing project a new project was sought. While there were many requirements for the project, by far the most important was that the project had to allow the students to focus on the design of experimental procedures. Operationally, this meant that the project had to be straightforward and have well defined relationships between the variables. The only other firm requirement was that the project must not be too costly. Beyond these requirements, potential projects should be interesting, hands on, and challenging.

Because many cadets are interested in parachuting, an experiment was chosen that required cadets to determine the coefficient of drag for a small, dollar-store paratrooper. This task is fairly straightforward using the concepts of terminal velocity and dimensional analysis and is inexpensive enough for each group of cadets to be given their own test specimen.

With a promising experiment identified, an abbreviated design process was introduced to the cadets. In its most basic form, design includes four phases, (1) plan for the design process, (2) develop engineering specifications, (3) develop concepts, (4) develop products. ${ }^{2}$ Although design is unquestionably an iterative process, emphasis was not placed on the iterative nature of design. Although much of the problem definition phase was given to the cadets, they did have to develop a schedule and try to find a time when all of the group members could get together. During the second phase, develop engineering specifications, cadets were required to identify governing equations and measures of goodness. Measures of goodness focused on minimizing uncertainty in the dependent variable and the availability of required resources. The third phase, concept generation, consisted primarily of modifications of a basic freefall procedure. There were two basic ways that the procedure could be modified, the cadets could modify the operating conditions or the techniques used to measure the parameters of interest. Modifications of the operating conditions consisted of altering the weight of the paratrooper, the height from which it was dropped, or even the fluid in which it was dropped. Additionally, alternative measurement techniques were considered. The evaluation of these concepts was accomplished by objective and subjective means. The uncertainty of the dependent variable was calculated using the following equation:

$$
U_{C_{D}}=\sqrt{\left(\frac{\partial C_{D}}{\partial x_{1}} U_{x_{1}}\right)^{2}+\left(\frac{\partial C_{D}}{\partial x_{2}} U_{x_{2}}\right)^{2}+\ldots\left(\frac{\partial C_{D}}{\partial x_{n}} U_{x_{n}}\right)^{2}}
$$

where $\mathrm{U}$ is the uncertainty in a variable, and $\mathrm{x}_{1}, \mathrm{x}_{2}, \ldots \mathrm{x}_{\mathrm{n}}$ are the independent variables. ${ }^{3}$. While calculating the uncertainty in the dependent variable gives an unmistakable measure of the goodness of a procedure, cadets are also required to make a subjective assessment of the feasibility of a procedure that addresses whether or not they can obtain measurement devises that 
can deliver the precision that they require. Finally, during the fourth phase, product generation, the cadets performed the procedure that they found to be the best. They refined the uncertainty calculations and calculated the coefficient of drag for the paratrooper.

\section{Results}

To determine the effectiveness of the paratrooper experimental design, two techniques were employed. First, an anonymous online survey was administered to about half of the 160 cadets enrolled in the fall semester. Second, anecdotal evidence was gathered from each faculty member to identify any exceptional submissions.

The survey provided valuable insight into cadet perceptions of the project. The data indicate that $89 \%$ of cadets either agreed or strongly agreed that the project was interesting. This is not terribly surprising given that many of the cadets had recently participated in Airborne school where they learn how to parachute. Roughly $80 \%$ of the cadets either agreed or strongly agreed that the project was straightforward. Some open ended survey question responses confirmed this opinion as cadets commented that their analyses was supported by common sense and intuition. These results are encouraging because cadets tend to work harder and learn more from projects that they find interesting and that are not overly complex.

Additional survey questions focused on how hard the cadets worked and how much they enjoyed doing the work. Figure 1 reports the results of these questions.

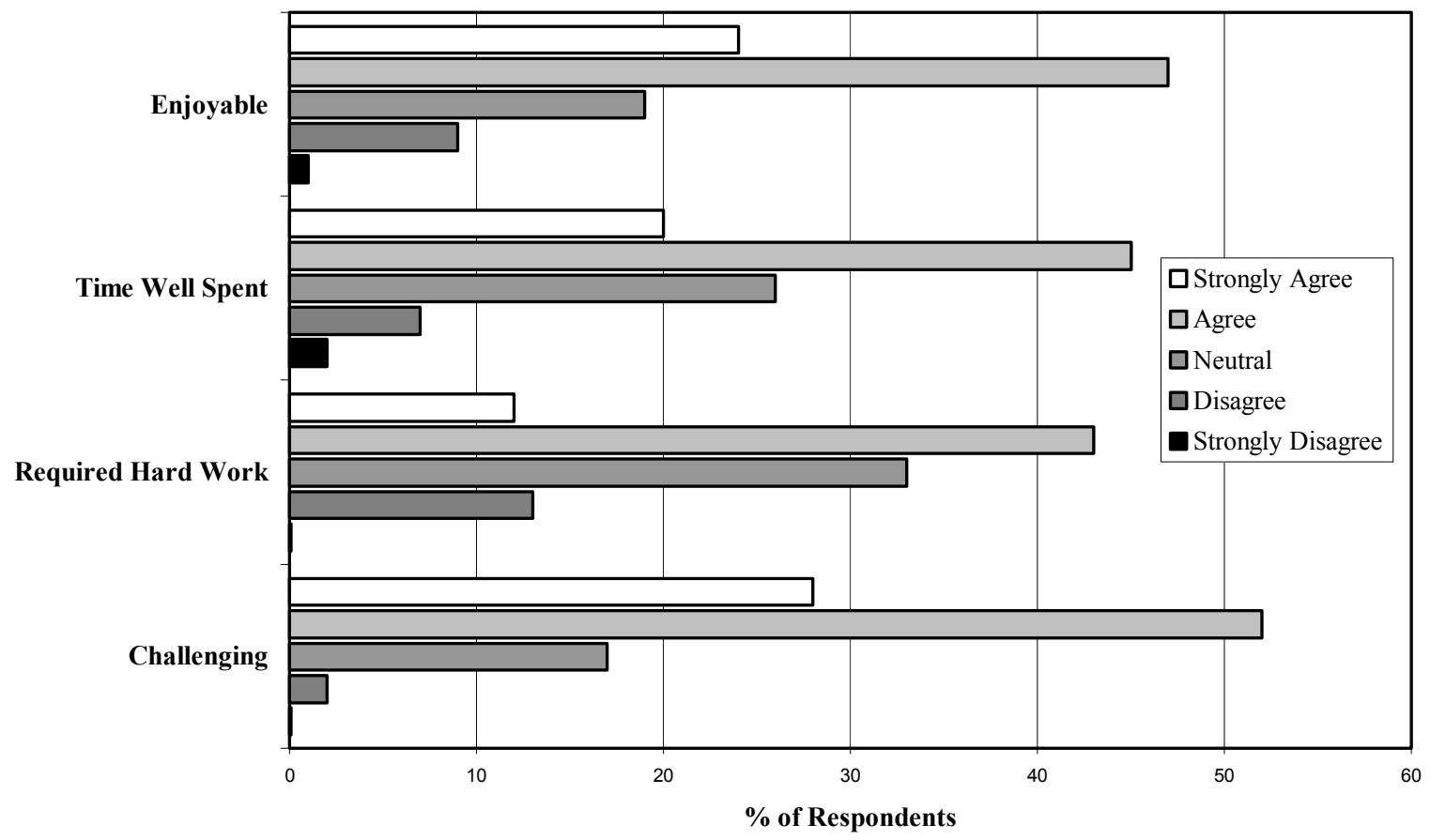

Figure 1: Cadet Perceptions of the Paratrooper Design of Experiment

From Figure 1 it can be seen that, by and large, cadets enjoyed working on the project even though it was challenging and required hard work.

While the previous results were encouraging, if the project failed to illustrate how to design experimental procedures, then the project was a failure. Figure 2 reports the students perceptions of whether or not they believed that they accomplished this objective. 
From Figure 2, it can be seen that roughly two-thirds of the cadets felt that they had developed the ability to design and conduct experiments as a result of this project. Unfortunately, only nine percent strongly agreed with this statement. This indicates that lot of them learned a little while only a few learned a lot. Further supporting this conclusion is the relatively small group, only ten percent, who felt that they had not developed the ability to design and conduct experiments. The most likely explanation for this is seen in some of the open-ended survey question responses. Several cadets stated that the uncertainty analysis calculations became a bit overwhelming. Additionally, some groups were dominated by one individual who did most of the work and merely explained it to the other group members.

The open ended survey questions

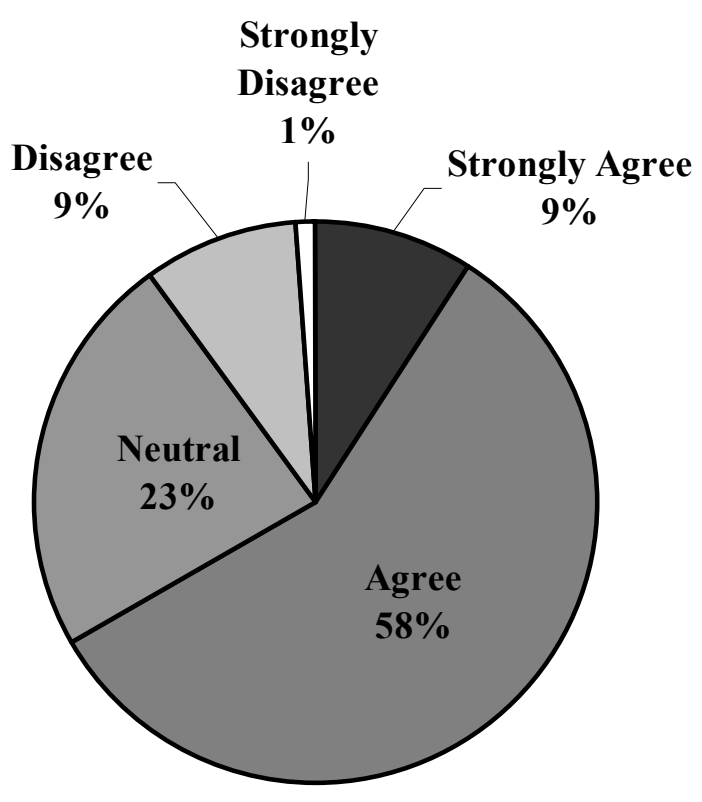

Figure 2: Did the cadets feel that they had developed the ability to design and conduct an experiment?

also yielded important insight into the effectiveness of the project. Many cadets cited a lack of guidance and a lack of direction. The project guidance was intentionally vague in some areas and did not constrain the cadets in any way. For some cadets, this was more than a little unnerving. Many though enjoyed the freedom that they were given in the project. Other comments from the open ended questions addressed that working in groups was either beneficial or detrimental depending on the members of the group. The most often comment seen in these responses dealt with the time required to perform the analysis, design the experimental procedure, conduct the experiment and write the report. Most felt that the project was time consuming. However, as was previously shown in Figure 1, many felt that the time was well spent and that they enjoyed doing the work.

While the survey data was positive, some of the actual cadet projects provided the most encouraging support for the project. For instance, while most groups dropped their paratrooper from various heights around campus, one group decided to don scuba gear and drop their paratrooper in the indoor pool. This particular group faced many additional obstacles, not he least of which was that the paratrooper was naturally buoyant. Nevertheless, they put in effort far beyond what was required because they were excited and interested by the project. In another instance, a group prepared a very entertaining briefing that was set to music and filled with digital snapshots of their trials and tribulations. Both groups fulfilled the requirement to design an experimental procedure and then exceeded the standard by having a lot fun and showing creativity.

\section{Recommendations}

Although much of the data was promising, there are many aspects of the project that can be improved. For instance, many cadets did not consider that the coefficient of drag that they had calculated does not necessarily correspond to the coefficient of drag of a real paratrooper. 
This project was issued during a block of instruction that focuses on dimensional analysis and similitude and future iterations of this projects will have cadets address whether or not this experiment accurately models the free fall of a real paratrooper. Additionally, while most cadets enjoyed the lack of constraints placed on the project, the analysis and report requirements will be more clearly defined to ensure that all of the groups are on track.

Another modification to the project will attempt to both increase the number of students who learn a lot about how to design an experimental procedure and decrease the amount of time spent performing monotonous uncertainty analysis calculations. Because all cadets are issued and have used MathCad in previous math classes, it's use will be required to perform the uncertainty calculations. By constructing one spreadsheet and using it over and over, cadets will be able to quickly and accurately perform the required uncertainty analysis calculations in a relatively short amount of time. This will greatly assist the cadets in trying to determine which variables and which uncertainties contribute the most to the overall uncertainty. The cadets will be required to plot sensitivities of the overall uncertainty to several independent variables. One concern with requiring the use of MathCad is that many cadets have had frustrating experiences with it in previous classes. To ensure that this project does not turn into an exasperating MathCad exercise, all cadets will be required to turn in one assignment earlier in the semester where they will construct a spreadsheet that will greatly assist them on this project. Additionally, a tutorial will be placed on the course website to address common problems that they may encounter.

\section{Conclusions}

The primary objective of this project was to develop the cadet's ability to design an experiment. Cadets generated different experimental procedures, evaluated them using uncertainty analysis and subjective evaluations regarding the availability of required resources, selected the best procedure and conducted the experiment. Most cadets felt that the project was beneficial although only a small percentage agreed strongly with statements regarding the effectiveness of the project. A large percentage of the cadets enjoyed the project and found it to be interesting and time well spent. Recommendations for improving the project focus on automating the uncertainty analysis so that key relationships are highlighted more clearly. With this and other minor modifications, this project will undoubtedly be one that will influence how cadets perform experiments in follow on coursework.

\section{References}

\footnotetext{
${ }^{1}$ Accreditation Board for Engineering and Technology, Inc. 2001-2002 Criteria for Accrediting Engineering Programs. Baltimore: 2001.

${ }^{2}$ Ullman, D. The Mechanical Engineering Design Process. Boston: McGraw-Hill, 1997.

${ }^{3}$ Crowe, C., Roberson, J., and Elger, D. Engineering Fluid Mechanics. New York: John Wiley \& Sons, 2001.
} 